\title{
Tackling ethical issues in health technology assessment: A proposed framework
}

\author{
Amanda Burls \\ University of Oxford and Oxfordshire Primary Care Trust \\ Lorraine Caron \\ Institut National d'Excellence en Santé et en Services Sociaux (INESSS) \\ Ghislaine Cleret de Langavant \\ Université de Montréal and Commissaire à la santé et au bien-être (Québec) \\ Wybo Dondorp \\ University Maastricht \\ Christa Harstall \\ Institute of Health Economics
}

Ela Pathak-Sen

Commotion UK

\section{Bjørn Hofmann}

University of Oslo; Norwegian Knowledge Center for the Health Services; and University College of Gjøvik

Objectives: Values are intrinsic to the use of health technology assessments (HTAs) in health policy, but neglecting value assumptions in HTA makes their results appear more robust or normatively neutral than may be the case. Results of a 2003 survey by the International Network of Agencies for Health Technology Assessment (INAHTA) revealed the existence of disparate methods for making values and ethical issues explicit when conducting HTA.

Methods: An Ethics Working Group, with representation from sixteen agencies, was established to develop a framework for addressing ethical issues in HTA. Using an iterative approach, with email exchanges and face-to-face workshops, a report on Handling Ethical Issues was produced.

Results: This study describes the development process and the agreed upon framework for reflexive ethical analysis that aims to uncover and explore the ethical implications of

We acknowledge the other members of the INAHTA/HTAi Ethics Working Group who contributed to the development of the report on Handling Ethical Issues, which forms the basis for this paper: Kerstin Hagenfeldt (Chair), SBU, Sweden; Svend Andersen, Centre for Bioethics (representing DACEHTA), Denmark; John Gabbay, NCCHTA, UK; Marta Lopez-Argumedo, OSTEBA, Spain; Hussein Noorani, CADTH, Canada. A special acknowledgement to Ken Bond, Research Associate, IHE, Canada for assisting with the writing and editing of the final version. Partial funding support was provided by the International Network of Agencies for Health Technology Assessment (INAHTA) and Health Technology Assessment International (HTAi) for face-to-face workshops. 
technologies through an integrated, context-sensitive approach and situates the proposed framework within previous work in the development of ethics analysis in HTA.

Conclusions: It is important that methodological approaches to address ethical reflection in HTA be integrative and context sensitive. The question-based approach described and recommended here is meant to elicit this type of reflection in a way that can be used by HTA agencies. The questions proposed are considered only as a starting point for handling ethics issues, but their use would represent a significant improvement over much of the existing practice.

Keywords: Ethics, Health technology assessment, Bioethics, Research design, Ethical framework

Health technology assessment (HTA) is necessarily valueladen $(13 ; 16 ; 18 ; 36)$. Ethical issues and decisions arise throughout the HTA process with values and normative assumptions underlying the development of a technology, its selection and prioritization for evaluation, the framing and methods used in an HTA, the identification and consideration of the values of stakeholders, and the evaluation of the ethical consequences of the implementation of a particular technology. There are always moral issues, although these are often not recognized explicitly. Even the most limited interpretation of HTA, as a purely technical tool for estimating the net benefit of technologies in a health maximizing system, involves value judgments (35), for example, what is the relative utility of different outcomes? Whose preferences and utilities should count? Which end points are suitable?

A distinction is often drawn between the "scientific" side of HTA—obtaining, critically appraising, and synthesizing research evidence-and the "value" side-making recommendations or decisions about whether and how a technology should be used-taking into account ethical and contextual considerations (often designated as "assessment" and "appraisal," respectively) (11). While this scientific/value distinction may be helpful for defining roles, it does not, and should not, preclude ethical analysis in the assessment process $(27 ; 37)$.

Ethical issues may be not addressed explicitly; may be analyzed as an adjunct to assessment; or considered explicitly as an integral part of the process. This has moral consequences because the approach chosen can influence findings and how these are interpreted or applied. We argue that ethical analysis should be integral to HTA and give suggestions about how this might be done.

As HTA inevitably is value-laden, ethical analysis aims to make these values explicit and to explore ethical consequences such that decisions can be fully informed. By integrating ethical analysis into assessment, findings become more relevant and may help decision makers decide "what do these conclusions mean in this particular situation?" Ethical analysis is reflexive in nature, seeking to identify and understand the morally relevant aspects of a situation rather than telling people what to do. Because even basic decisions may reflect implicit values, it can be helpful for ethical issues to be analyzed early in the assessment process. The values of the assessors may also influence this analysis; therefore, it is important for assessors to be transparent about their ethical position and its implications such that decision makers, who may not share their values or context, can interpret the findings (2). Ethical analysis in HTA should avoid being directive-it should make normative issues explicit and discuss the acceptability of policy options, while acknowledging that decisions and actions on recommendations are the prerogative of decision makers.

In 2003, the International Network of Agencies for Health Technology Assessment (INAHTA) surveyed its members about how they deal with ethical issues in HTA (20). The questionnaire was sent by email to INAHTA member agencies whose representatives were self-identified as having a keen interest in including ethics in their assessments. Representatives were asked to provide the following information: (i) a description of HTA product(s) which included ethical analysis (title of the report, date, research question[s], characteristics of the intervention, target population, methodology used to approach the ethical subject); (ii) the stage in the HTA process at which ethical considerations were raised and discussed; (iii) if there is a standardized ethics process used for all HTA products used by the agency; and (iv) who is responsible for the ethics component in a report (i.e., what knowledge do they have with respect to identifying and analyzing ethical issues). The results of the survey indicated that there was great variation in how ethical issues in HTA were handled. Prompted by these results, the INAHTA Board proposed the establishment of a Working Group on Ethical Issues. The Ethics Working Group comprised representatives of the HTA agencies that replied to the survey and represented eleven countries and sixteen HTA agencies and included bioethicists, policy makers, doctors, researchers, HTA producers, and agency managers. (Note: The INAHTA Ethics Working Group became a joint initiative with HTAi in 2005). The INAHTA Board developed terms of reference that set out the particular questions that the newly formed Working Group was to address (Table 1).

This study outlines an agreed framework for integrating explicit consideration of ethical issues into HTA based on the framework for ethical analysis developed by the INAHTA Ethics Working Group in response to these questions. 
Burls et al.

Table 1. Questions Proposed by the INAHTA Board to Be Addressed by the Ethics Working Group

1. Can there be a procedure for handling ethical issues concerning technologies being assessed?

2. If yes, what would such a procedure look like?

3. If not, why not, and what else can be done to assure good quality of the assessment of the ethical aspects of a technology?

4. What kind of ethical issues (e.g., consequences, duties, human rights, ethical principles) and questions are relevant with respect to a given technology?

5. How far should HTA go in displaying values involved in the HTA process itself? Highlighting relationships between knowledge and norms? Making recommendations with respect to ethical issues?

6. What is the relevance of addressing ethical issues with respect to achieving a successful dissemination? With respect to professionals? With respect to health policy?

7. What kinds of methods might be used to tackle these kinds of issues in an HTA, and how might INAHTA help to agree on appropriate methodologies and quality checks?

8. What can be done to find or develop skills that would be required by HTA agencies undertaking ethical analyses?

INAHTA, International Network of Agencies for Health Technology Assessment; HTA, health technology assessment.

\section{METHODS}

A chair was appointed to lead and coordinate a work assignment of the Ethics Working Group. The Ethics Working Group members, based on their interests and expertise, selected which questions posed by the INAHTA Board they wished to work on. A lead was assigned for each question and was responsible for synthesizing the responses of their small group. Draft papers addressing each question were developed collaboratively by email. These were then synthesized in an iterative process into a single document that was discussed by the wider group during the HTAi meeting in 2005 and then submitted to the INAHTA Board (21). The consideration by the Ethics Working Group of the ethicsrelated questions posed by the INAHTA Board led to the development of the approach described below.

\section{RESULTS}

\section{Possible Methods of Ethical Analysis}

There are many possible methods of ethical analysis, ranging from deductivist $(1 ; 5 ; 12)$ to contextualist $(3 ; 7 ; 16 ; 26)$ approaches. In the literature, several authors have reflected on the strengths and weaknesses of these different approaches with regard to using them in the context of HTA $(2 ; 13 ; 25 ; 36)$. After discussing the use of these methods and their strengths and weaknesses, the Working Group concluded that no single ethical method is likely to be sufficient. However, as the ethical implications of a technology are intimately linked to the context of its development, uptake, and use, approaches to integrating ethical reflection into HTA must be context sensitive. Authors have proposed different ways of achieving this, including reflexivity in the undertaking of assessments to recognize values involved in the framing of HTA questions (16;17), historical/social analysis to reveal the values underlying the development of a technology, and formal identification and analysis of stakeholder values, as part of an interactive HTA approach $(30 ; 31)$ or other forms of public involvement (24). Looking for an approach that would allow reconciling this context sensitivity with a practical frame- work to be used in HTA agencies, the Working Group found one proposal to be the most promising. This "axiological" approach aims to elicit ethical reflection by highlighting overt and covert value issues through a nonexhaustive selection of targeted questions (16), the questions to be addressed in the HTA bringing to the fore value issues of process, the technology, its implementation and use, its assessment, and its stakeholders.

\section{Questions to Help Structure Consideration of Ethical Issues}

By explicitly addressing the following questions (many derived from Hofmann) (16) during the HTA process, important ethical dimensions will be revealed (Table 2). Examples of how the questions can relate to HTA are provided and are elaborated upon in greater detail than was done in Hofmann's original proposal. These questions are not exhaustive but offer a starting point for reflecting on the possible ethical implications of an HTA. The later questions, those about the morally relevant consequences of the technology, are likely to be the most important. In addition to using this framework to clarify the ethically relevant features of a technology, it is important to make explicit the values of those doing the assessment and the interests of those involved in its application.

Q1. Why was this technology selected for assessment? The process of identifying and selecting areas for HTA has ethical dimensions. Priorities can be affected by who is involved in prioritization. A system that is mainly driven by industry, for example, has the potential to move high cost technologies onto the agenda and displace more cost-effective technologies that do not have a sponsor. It is important that the process be explicit, systematic, and transparent (28). Involving all stakeholders in prioritization can help balance conflicting interests and may help the dissemination and implementation of results.

Q2. At what point in a technology's development should it be assessed? Technologies assessed too early may appear ineffective, while technologies assessed too late may either have become established to an 
Table 2. Questions to Motivate Ethical Reflection and Analysis in HTA

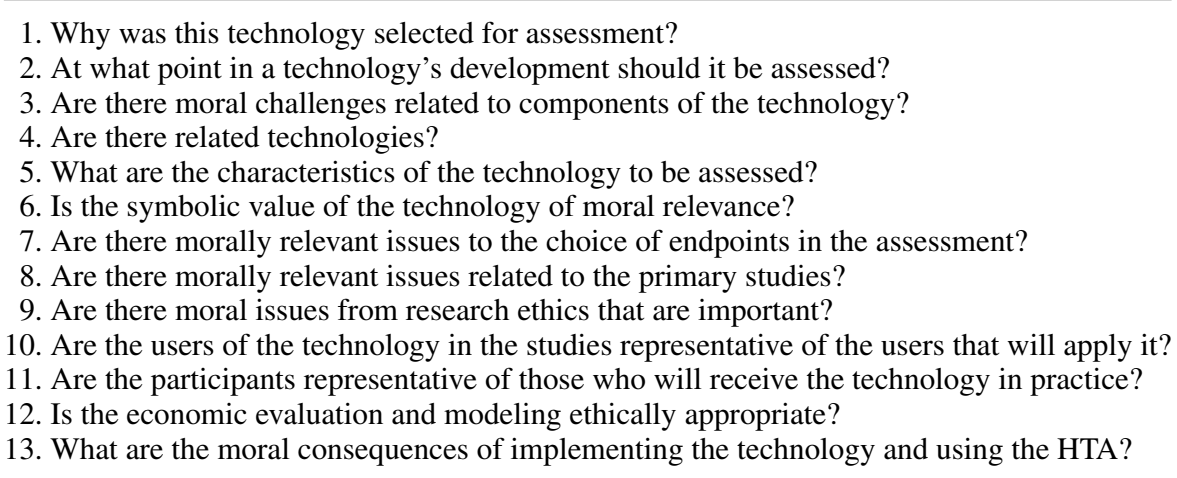

HTA, health technology assessment.

inappropriate degree or, when effective, not have fulfilled their potential for health gain due to inadequate diffusion into practice. Hence, the timing of an assessment is morally relevant.

\section{Q3. Are there moral challenges related to com-} ponents of the technology? If a technology uses or depends upon other technologies, it is important to consider the ethical issues related to these.

Q4. Are there related technologies? The assessment of a technology is always comparative. Many existing technologies have not been properly assessed. Failure to assess related technologies means decision makers may not have all the information they need about alternatives or interactions and can distort the findings and consequent decisions. Moreover, related technologies may have been assessed and their ethical analysis may be of relevance in assessing this particular technology.

Q5. What is the characteristic of the technology to be assessed? A technology is characterized by its function or purpose (19) and it is important to highlight morally relevant values related to these. An example is pediatric cochlear implants that help children hear. Improving hearing is valued differently depending on whether deafness is perceived as a pathological disorder or as a feature of a specific cultural minority (29;36). A technology may also establish responsibilities within healthcare systems. For example, since the advent of prenatal tests, the healthcare system is often held responsible when such tests are not performed or fail.

Q6. Is the symbolic value of the technology of moral relevance? Technologies tend to have status, the perception of which can differ among the various stakeholders. For example, cardiac lasers may have a higher symbolic value than crutches or a service may be highly valued as a symbol of societal solidarity even when it is clinically ineffective. Symbolic value can be of moral relevance to an HTA.

Q7. Are there morally relevant issues related to the choice of endpoints in the assessment? The choice of endpoints is a matter of value. For example, is the aim of a technology to reduce mortality, increase functional status, decrease morbidity, or increase quality of life? At what point does an increase in life expectancy compensate reduced quality of life or vice versa?

Q8. Are there morally relevant issues related to the primary studies? The quality and type of studies included in an HTA may be of moral relevance. For example, are there moral implications of methodological norms, which focus on internal validity? Typically, methodologically weaker study designs are prone to overestimate a technology's effectiveness $(23 ; 33)$. By including these in an HTA some argue that a fuller picture is obtained. However, could the inclusion of these study designs encourage sponsors to undertake research of inadequate design? What if the result becomes statistically significant if a "borderline study" is included? Or if studies on a technology are suggestive of benefit but do not reach conventional levels of statistically significance and the technology is the only specific treatment available, like riluzole in motor neurone disease $(32 ; 34)$ ? Should diagnostic technologies be evaluated on the basis of treatment outcomes or simply on diagnostic accuracy? These methodological questions are central to continuing disputes about HTAs and are of moral relevance.

Q9. Are there moral issues from research ethics that are important? For research to be ethical it needs to use valid methods and respect the welfare and dignity of participants. Many studies do not report morally relevant issues such as financial support, conflicts of interest, publication biases, or justification of sample size (38) which can affect the findings of studies $(4 ; 10)$. Furthermore, there is ongoing debate over the interpretation of clinical equipoise and what constitutes a suitable reference group or comparator in clinical studies. Should the ethics of primary studies be evaluated when undertaking a systematic review and, if so, what aspect? For example, should scientifically robust studies, which do not raise significant ethical issues but which were not approved by an ethics committee or institutional 
review board, be included in HTAs? How unethical do studies have to be before they are excluded?

Q10. Are the users of the technology in the studies representative of the users that will apply it? The results obtained by experts or enthusiasts using a technology may not be the same as those achieved in routine practice. This may be morally relevant if the technology is used in a context different from the one in which it was tested.

Q11. Are the participants representative of those who will receive the technology in practice? The degree to which one can generalize from a study's participant population to the population for whom a decision is being made is known as external validity and may be morally relevant, e.g., do both groups value the outcomes in the same way?

Q12. Is the economic evaluation and modeling ethically appropriate? The particular type of economic evaluation undertaken in an HTA may have ethical dimensions. For example, cost-utility analyses require different outcomes to be transformed into a common metric and carry implicit ethical assumptions (14). Quality-adjusted life-years (QALYs), for example, assume that it is possible to trade off quality and quantity of life. Do all QALYs have the same value, for example, are they worth the same at the end of life? If incremental cost-effectiveness ratios from cost-utility analyses are used to rank or prioritize treatments or to determine whether they meet a certain willingness-to-pay threshold, then this is fundamentally a utilitarian approach. The economic model used to assess cost-effectiveness also needs to be considered, for example, what values underlie the construction and use of the model? How relevant and reasonable are the parameters and structure used? What consequences do they have?

Q13. What are the moral consequences of implementing the technology and using the HTA?

Even though it is appropriate that decisions on access to a technology should be in the hands of the decision makers, the consequences of use of the technology and rationing should be explicit and may aid decision makers appreciate the implications of their decisions.

\section{Other Considerations}

A distinction can be made between personal, professional, social, and political ethics. For example, a physician has professional ethical responsibilities (such as a duty of care to his or her patient) whereas healthcare organizations typically embody political ethics, for example, in their control of access to healthcare. Therefore, ethical questions can differ depending on context and the responsibilities of the stakeholders. The challenge lies in identifying the normative basis of these ethical perspectives. All levels need to be considered and are not mutually exclusive, for example, at the professional level the application of a technology depends on clinical context which overlaps with the political aspects of legislation and access to care.

Ethical reflexivity may also go beyond looking at the evidence and its synthesis to include procedural features like encouraging greater public participation and increasing the transparency of decision making (22). This participatory or interactive approach $(29 ; 30)$ can help ensure inclusion of aspects otherwise easily overlooked and relevant to decisions.

\section{DISCUSSION}

There are many suggested approaches for handling ethical issues in HTA, and this plurality can be confusing. The aim of the INAHTA meeting was to recognize the legitimacy of this plurality and to involve ethicists working in HTA to come up with a common starting point (although not a common analytic approach) for ethics analysis. Through a combination of face-to-face meetings and email conversation, the Working Group agreed upon a set of thirteen questions that could serve this purpose.

Hofmann (16) has presented a list of thirty-three "morally relevant questions" that poses questions with respect to the general moral issues related to health technology and the HTA process. Ten of the thirteen questions used here have equivalents with Hofmann's questions and particular approaches or issues. For example, Q13 expresses a utilitarian approach and Q4 a casuistic approach, while Q6 considers the sociocultural embeddedness of the technology and its symbolic value within that context. The questions that were accepted by the working group that were not a part of Hofmann's questions (Q10-12) concern ethical issues in the methodological choices made in conducting an HTA rather than with the implementation of the technology per se, thus addressing and potentially highlighting the ethical assumptions of the clinical and economic evaluation of the technology.

The EUnetHTA Core Model (9) proposes an approach to ethics analysis similar to the one developed here in holding that a set of questions constitute the "core" of an ethics analysis, that is, form a minimum first step for ethics analysis. Indeed, as one early reviewer of this study maintained, defining the core questions was the basis for the entire EUnetHTA project, which also relied on Hofmann's (16) work. The authors of the ethics section of the EUnetHTA document acknowledge that the approaches currently used and described in the document had been identified and defined by the INAHTA Ethics Working Group (9). However, there are significant differences in both the development of the framework and in the framework itself, differences that may have an impact on its broader usefulness. The EUnetHTA Core Model lists a total of fifteen questions, only four of which are drawn from Hofmann's list. One plausible reason for this difference is that the INAHTA group involved a broader cross-section of ethics researchers by including ethicists from outside the European network. This broader 
perspective brought to the table an enriched debate that resulted in a smaller number of questions that were considered relevant from this diversity of viewpoints.

The EUnetHTA Core Model went somewhat further to recommend several approaches that might be used beyond the core questions. In contrast, the approach proposed here remains silent on the methods to be used once these questions have been addressed, preferring instead to allow local context and expertise to determine what next steps should be taken. In this, the authors of this study agree with the authors of the EUnetHTA report that the most suitable method locally must be chosen to suit the resources available, the HTA topic, position of the HTA organization in the healthcare system of the country, and the competencies of those performing the ethics analysis (9). Local variation of methods and procedures is not necessarily problematic as long as transparent documentation is provided.

Values such as public accountability, quality of care, and justice are intrinsic to the use of HTAs in health policy. HTA reports in contrast aim for scientific objectivity and neutrality. The need for scientific legitimacy in HTA (30) has contributed to the neglect of the ethical dimensions, such as the failure to acknowledge that data are inevitably value-laden, for example, the choice of instrument for measuring quality of life or costs identified in an economic evaluation (14). When assumptions are not explicit, HTA outcomes appear more robust or normatively "neutral" than they really are. In a recent review, only a small minority of HTAs included an explicit discussion of the ethical aspects of the technology under consideration (8). This may be due to the lack of a conceptual and methodological framework.

It is proposed that the above questions are but the first step toward developing a framework. One method for operationalizing this approach could be for ethical issues to be discussed with the relevant decision makers at the topic refinement phase of each HTA, having first mapped out who the stakeholders are, including those who are not immediately apparent. Morally relevant questions identified a priori could be explicitly considered during assessment. Following the topic refinement phase, the project team could identify a person responsible for the ethical analysis. The literature search could seek information on ethical aspects of the technology and related technologies (6). Content experts within the team can help identify ethical dilemmas that emerge from the development and use of the specific technology. Study findings should include a qualitative analysis of the relevant ethical issues and resultant policy implications and consideration of the context of the healthcare system for which the assessment is being undertaken. Exploration of stakeholders' values through surveys, interviews, and workshops may be necessary where information is lacking.

In a recent article, Grunwald (13) made a helpful distinction between an HTA where there is a pre-existing normative consensus (the article explores criteria for this) and situations where a consensus does not exist. In the former, HTA is able to give orientation without extensive ethical analysis. In the latter, the normative issues surrounding the introduction of the technology require explicit analysis and, if possible, resolution. Examples are the debates on the moral acceptability of preimplantation genetic screening or the medical use of human embryonic stem cells. An important element of the role of ethics in HTA is to judge whether the situation under consideration belongs to the "business as usual" or "moral conflict" category and this could be determined in the above process. Axiological approaches have also become more widely known and accepted in the bioethics literature (15).

In situations of moral conflict, ethical analysis should aim at providing policy makers with a thorough analysis of the relevant dimensions of the problem, or contribute to resolution by means of interactive approaches $(29 ; 30)$. Failure to consider ethical issues explicitly can make HTAs less useful by failing to take into account the context of a decision, overlooking important issues or consequences that might lead to different findings or decisions, and inadequately representing community values (which may, in turn, present a barrier to dissemination or implementation).

\section{CONCLUSIONS}

There is no way to avoid ethical issues when assessing technologies. If these are ignored, embedded values may not be transparent and HTAs may be less useful for decision makers. It is unlikely that a single method will reveal all ethical issues; however, it is important that methodological approaches to address ethical reflection in HTA be integrative and context sensitive. The questions-approach described and recommended here is meant to elicit this type of reflection The questions proposed are meant as a starting point; they are neither exhaustive nor sufficient, but their use would represent a significant improvement over much of the existing current practice. We hope the framework will guide HTA organizations on how they might undertake ethical analysis, encourage them to do so, and stimulate debate.

\section{CONTACT INFORMATION}

Amanda Burls, MB BS, MSc (Amanda.Burls@phc.ox. ac.uk), Director of Postgraduate Programmes in EvidenceBased Health Care, Department of Primary Health Care, University of Oxford, Roosevelt Drive, Headington, Oxford, Oxfordshire OX3 7LF, UK; Honorary Consultant in Public Health, Department of Public Health, Oxfordshire Primary Care Trust, Jubilee House, 5510 John Smith Drive, Oxford Business Park South, Cowley, Oxford, Oxfordshire OX4 2LH, UK

Lorraine Caron, PhD (lorraine.caron@inesss.qc.ca), Researcher, Institut National d'Excellence en Santé et en Services Sociaux (INESSS), 2021 avenue Union, bureau 10.083, Montréal, Québec, Canada H3A 2S9 
Ghislaine Cleret de Langavant, $\mathrm{PhD}$ (ghislaine. delangavant@csbe.gouv.qc.ca), Associate Professor, Department of Health Administration (DASUM), Université de Montréal, C.P. 6128, succ. Centre-ville, Montréal, Québec, Canada H3C 3J7; Deputy Health Commissioner (responsible for ethics), Commissaire à la santé et au bien-être (Québec), 2021 Union street, \# 870, Montréal, Québec, Canada H3A $2 \mathrm{~S} 9$

Wybo Dondorp, PhD (w.dondorp@ maastrichtuniversity.nl), Senior Research Fellow, Research School CAPHRI and Department of Health Ethics and Society, University Maastricht, Universiteitssingel 40, 6229 ER Maastricht, The Netherlands Christa Harstall, BScMLS, MHSA (charstall@ihe.ca), Director, Department of Health Technology Assessment, Institute of Health Economics, 1200 - 10405 Jasper Avenue, Edmonton, Alberta, Canada T5J 3N4

Ela Pathak-Sen, BA, BEd, MBA (ela@ commotiouk.com), Director, Department of Patient \& Public Involvement, Commotion UK, Culvert Cottage, Kingsmill Lane, Painswick, Gloucestershire GL6 6RT, UK

Bjørn Hofmann, MSc, PhD (b.m.hofmann@medisin. uio.no), Professor, Center for Medical Ethics, University of Oslo, P.O. Box 1130, Blindern, N-0318 Oslo, Norway; Researcher, Norwegian Knowledge Center for the Health Services, P.O. Box 7004, St Olavs plass, N-0130 Oslo, Norway; University College of Gjøvik, P.O. Box 1, N-2802 Gjøvik, Norway

\section{CONFLICT OF INTEREST}

Christa Harstall's institution has received support for travel to meetings for the study from unidentified source and Ela Pathak-Sen's institution has received funding for consultancy from NICE. The other authors report they have no potential conflicts of interest.

\section{REFERENCES}

1. Beauchamp TL, Childress TF. Principles of biomedical ethics. 6th ed. New York, NY: Oxford University Press; 2009.

2. Blancquaert I, Cleret de Langavant G, Bouchard L. L'évaluation des technologies de la santé à l'ère de la génomique. Le défi de la complexité. Ruptures, revue transdisciplinaire en santé. 2002;9:22-38.

3. Cleret de Langavant G. Bioéthique: Méthode et complexité. Québec: Les Presses de 1'Université du Québec; 2001.

4. Coyne JC. Cochrane reviews $\mathrm{v}$ industry supported metaanalyses: We should read all reviews with caution. BMJ. 2006;333:916.

5. Daniels N. Wide reflective equilibrium in practice. In: Daniels $\mathrm{N}$, ed. Justice and justification. Reflective equilibrium in theory and practice. New York: Cambridge University Press; 1996.

6. Decker M. The role of ethics in interdisciplinary technology assessment. Poiesis Prax. 2004;2:139-156.

7. Droste S, Dintsios CM, Gerber A. Information on ethical issues in health technology assessment: How and where to find them. Int J Technol Assess Health Care. 2010;26:441-449.
8. Droste S, Gerhardus A, Kollek R. Methoden zur Erfassung ethischer Aspekte und gesellschaftlicher Wertvorstellungen in Kurz-HTA-Berichten - eine internationale Bestandsaufnahme. Deutsche Agentur für Health Technology Assessment des Deutschen Instituts für Medizinische Dokumentation und Information (DAHTA@DIMDI), Köln, 2003.

9. EUnetHTA. HTA core model for medical and surgical interventions, v1.0r. Work Package 4. Helsinki: Finnish Office for HTA; 2008. http://www.eunethta.net/upload/WP4/Final\% 20Deliverables/HTA\%20Core\%20Mode1\%20for\%20Medical $\% 20$ and\%20Surgical\%20Interventions\%201\%200r.pdf (accessed February 4, 2011).

10. Friedberg M, Saffran B, Stinson TJ, Nelson W, Bennett CL. Evaluation of conflict of interest in economic analyses of new drugs used in oncology. JAMA. 1999;282:14531457.

11. Gallo P. Integrating ethical enquiry and health technology assessment: Limits and opportunities for efficiency and equity. Poiesis Prax. 2004;2:103-117.

12. Gillon R. Philosophical medical ethics. London: John Wiley \& Sons; 1986.

13. Grunwald A. The normative basis of (health) technology assessment and the role of ethical expertise. Poiesis Prax. 2004;2:175193.

14. Health Council of the Netherlands. Ethical aspects of costutility analysis. 4th National Ethics Councils in EU Countries, Amsterdam, December 22, 2004.

15. Hofmann B. Stuck in the middle: The many moral challenges with bariatric surgery. Am J Bioeth. 2010;10:3-11.

16. Hofmann B. Towards a procedure for integrating moral issues in HTA. Int J Technol Assess Health Care. 2005;21:312-318.

17. Hofmann B. On value-judgements and ethics in health technology assessment. Poiesis Prax. 2005;3:277-295.

18. Hofmann B. How ethics should be part of health technology assessment. Int J Technol Assess Health Care. 2008;24:423429.

19. Hofmann B. The technological invention of disease [thesis]. Oslo: University of Oslo Press; 2002.

20. INAHTA. Handling ethical issues in INAHTA agencies. INAHTA Newsletter, March 2003. www.inahta.org/upload/ Newsletters/Newsletter\%20Jan-Mar\%2003.pdf (accessed February 4,2011$)$.

21. INAHTA Ethics Working Group. INAHTA's Working Group on Handling Ethical Issues. Final Report, June 2005, pp. 121. www.inahta.org/upload/Final\%20report\%20Ethics\%20in\% 20HTA\%20Nov\%2007.pdf (accessed February 4, 2011).

22. Johri M, Lehoux P. The great escape? Prospects for regulating access to technology through health technology assessment. Intl J Technol Assess Health Care. 2003;19:179-193.

23. Juni P, Altman DG, Egger M. Systematic reviews in health care: Assessing the quality of controlled clinical trials. BMJ. 2001;323:42-46.

24. Lehoux P, Daudelin G, Demers-Payette O, Boivin A. Fostering deliberations about health innovation: What do we want to know from publics? Soc Sci Med. 2009;68:2002-2009.

25. Leventhal E, Brodin H, Gabbay J. The incorporation of ethics in health technology assessments - a new conceptual framework. A report for the NHS NCCHTA based on a Masters dissertation for an MSc in International Health Policy, University of Southampton, 2004. 
26. Leys M. Health technology assessment: The contribution of qualitative research. Intl J Technol Assess Health Care. 2003;19:317-329.

27. Molewijk AC, Stiggelbout AM, Otten W, Dupuis HM, Kievit J. Implicit normativity in evidence-based medicine: A plea for integrated empirical ethics research. Health Care Anal. 2003;11:69-92.

28. Oortwijn W. First things first: Priority setting for health technology assessment (dissertation). University of Amsterdam; 2000; 207.

29. Reuzel RPB. Interactive technology assessment of paediatric cochlear implantation. Poiesis Prax. 2004;2:119-137.

30. Reuzel RPB. Health Technology assessment and interactive evaluation. Different perspectives. Nijmegen University; 2001.

31. Reuzel R, van der Wilt GJ, ten have HA, de Vries Robbe PF. Reducing normative bias in health technology assessment: Interactive evaluation and casuistry. Med Health Care Philos 1999;2:255-263.

32. Sandercock J, Burls A, Hyde C, et al. Riluzole for motor neurone disease. More trials are needed. BMJ. 2001;322: 1305.

33. Schultz KF, Chalmers I, Hayes R, Altman DJ. Empirical evidence of bias. Dimensions of methodological quality associated with estimates of treatment effects in controlled trials. JAMA. 1995;273:408-412.

34. Stewart A, Sandercock J, Bryan S, et al. The clinical effectiveness and cost-effectiveness of riluzole for motor neurone disease: A rapid and systematic review. Health Technol Assess. 2001;5:1-97.

35. Strech D, Tilburt J. Value judgments in the analysis and synthesis of evidence. J Clin Epidemiol. 2008;61:521-524.

36. ten Have H. Ethical perspectives on health technology assessment. Intl J Technol Assess Health Care. 2004;20:71-76.

37. Van der Wilt GJ. Health technology assessment: Trying to bring empirical and ethical inquiry together. Poiesis Prax. 2004;2:195-206.

38. Weingarten MA, Paul M, Leibovici L. Assessing ethics of trials in systematic reviews. BMJ. 2004;328:1013-1014. 